\title{
Comparison of Blueberry (Vaccinium spp.) and Vitamin C via Antioxidative and Epigenetic Effects in \\ Human
}

\author{
Minju Kim ${ }^{1}$, Hyunkyung $\mathrm{Na}^{1}$, Hiroshi Kasai ${ }^{2}$, Kazuaki Kawai ${ }^{2}$, Yun-Shan $\mathrm{Li}^{2}$, Mihi Yang ${ }^{1}$ \\ ${ }^{1}$ Research Center for Cell Fate Control and College of Pharmacy, Sookmyung Women's University, Seoul, Korea, ${ }^{2}$ Department of Environmental \\ Oncology and Institute of Industrial Ecological Sciences, University of Occupational and Environmental Health, Kitakyushu, Japan
}

\begin{abstract}
Background: Chemopreventive effects and the underlying mechanisms of blueberry (Vaccinium spp.) are not clearly understood in human We hypothesized blueberry would work via antioxidative and epigenetic modulation, which is similar to vitamin C.

Methods: We performed a pilot and non-inferiority study in healthy young women $(\mathrm{n}=12)$, who consumed vitamin $\mathrm{C}(1 \mathrm{~g} / \mathrm{d})$ or 240 $\mathrm{mL}$ of blueberry juice (total polyphenols $300 \mathrm{mg}$ and proanthocyanidin $76 \mathrm{mg} / \mathrm{d}$ ) for 2 weeks. We analyzed 8-hydroxydeoxyguanosine $(8-\mathrm{OHdG})$ and malondialdehyde (MDA) levels in their urine, and global and specific DNA methylation at the NAD(P)H quinone oxidoreductase 1 (NQO1), methylenetetrahydrofolate reductase (MTHFR), or DNA methyltransferase 1 (DNMT1) genes in their blood. Results: Urinary 8-OHdG levels were reduced by blueberry consumption rather than by vitamin C. The methylation (\%) of the MTHFR was significantly decreased in blueberry-consumers and the antioxidant-susceptible subgroup, whose urinary MDA levels were decreased by the intervention. We also found a positive correlation between changes of urinary 8-OHdG and of DNA methylation at the MTHFR or the DNMT1 $(P<0.05)$. However, the genetic polymorphism of the MTHFR (C677T in exon 4) did not affect any above markers. Conclusions: Blueberry juice shows similar anti-oxidative or anti-premutagenic activity to vitamin $C$ and the potential as a methylation inhibitor for the MTHFR and the DNMT1 in human.
\end{abstract}

(J Cancer Prev 2017;22:174-181)

Key Words: Epigenetics, Blueberry, Oxidative stress, Vitamin C, MTHFR

\section{INTRODUCTION}

After the Human Genome Project, various omics technologies have been introduced to understand human diseases. Particularly, epigenetics or epigenomics have been emphasized to evaluate chemopreventive materials and to study the endpoints of environmental carcinogens. ${ }^{1}$ Epigenetic change has been demonstrated in response to a wide range of foods and nutrients and epigenetic status is emerging as a critical determinant of the response of the organism to the environment and its biological function and disease susceptibility. ${ }^{2}$

Growing evidence supports a role for reactive oxygen species (ROS) in epigenetic processes through the generation of oxidative stress. ${ }^{3}$ On the other hand, epigenetic processes are also influenced by a variety of dietary antioxidants. For example, maternal dietary antioxidant supplementation mitigated DNA methylation changes in the irradiated offspring. ${ }^{4}$

Antioxidative capacity, which leads to prevent genetic alterations by ROS, is a well-known characteristic of chemopreventive agents from natural resources for protection against oxidative stress-related diseases. ${ }^{5}$ Blueberry (Vaccinium spp.) belongs to the category of functional foods called "superfruits", which have the favorable combination of nutrient richness and antioxidant properties. ${ }^{6}$ Because of its nutrient rich ingredients, a series of studies on blueberry have been performed in many countries. It has been demonstrated that blueberry contains

Received September 9, 2017, Accepted September 11, 2017

Correspondence to: Mihi Yang

College of Pharmacy, Sookmyung Women's University, 100 Cheongpa-ro 47-gil, Yongsan-gu, Seoul 04310, Korea

Tel: +82-2-2077-7179, Fax: +82-2-710-9871, E-mail: myang@sm.ac.kr, ORCID: Mihi Yang, http://orcid.org/0000-0002-3683-2488

Copyright (C) 2017 Korean Society of Cancer Prevention

(c) This is an Open Access article distributed under the terms of the Creative Commons Attribution Non-Commercial License (http://creativecommons.org/icenses/by-n//4.0) which permits unrestricted non-commercial use, distribution, and reproduction in any medium, provided the original work is properly cited. 
anthocyanins, polyphenols including pterostilbene, and flavonoids and appears to have the highest antioxidant capacity among fruits and vegetables. ${ }^{7}$ Blueberry has shown a broad spectrum of biomedical functions in age-related oxidative stress, inflammatory responses, and diverse degenerative diseases. ${ }^{8}$

A variety of in vitro studies and animal studies have been performed to identify the chemopreventive mechanisms of blueberries. ${ }^{9}$ Beyond the antioxidant properties, blueberry anthocyanins induced G2/M cell cycle arrest and apoptosis of oral cancer cells by downregulating methylation of $\mathrm{p} 53 .{ }^{10}$ Moreover, anthocyanin-rich food including blueberry showed a significant role on vessel endothelium and had an enhanced effect in decreasing the risk of myocardial infarction. ${ }^{6}$ However, chemopreventive mechanisms of blueberry in human are not clarified yet. To support the roles of blueberries in animal and in vitro studies, robust evidences in human are encouraged to be investigated.

In the present pilot study, we evaluated anti-oxidative or anti-premutagenic and epigenetic effects of blueberry on healthy women. At first, we analyzed the effects of blueberry on oxidative stress biomarkers, i.e., urinary malondialdehyde (MDA), a typical biomarker for lipid oxidation, and 8-hydroxydeoxyguanosine (8-OHdG), a representative premutagenic biomarker. Secondly, we examined the effects of blueberry on global and specific DNA methylation at DNA methyltransferase 1 (DNMT $), \mathrm{NAD}(\mathrm{P}) \mathrm{H}$ quinone oxidoreductase 1 (NQOI), and methylenetetrahydrofolate reductase (MTHFR) genes to clarify the potential of blueberry or vitamin C. Thirdly, we studied the interaction between blueberry-induced epigenetic modulation and anti-oxidative or anti-premutagenic actions.

\section{MATERIALS AND METHODS}

\section{Clinical study}

This randomized non-inferiority study was designed to compare antioxidative effect of blueberry with that of vitamin C, a positive control for antioxidants, in human and performed with 12 healthy women at Sookmyung Women's University (Seoul, Korea: $\mathrm{n}=6$ for blueberry, $\mathrm{n}=6$ for vitamin C; Fig. $1 \mathrm{~A}$ ), who had similar menstrual patterns. The written informed consents were obtained from each of the study participants prior to their inclusion in the study. The allocation of vitamin $\mathrm{C}$ or blueberry was determined by lot, a simple random sampling. The six subjects consumed one tablet of vitamin C ( $1 \mathrm{~g} / \mathrm{d})$, which was the recommended daily dose of the manufacturer (Korea Eundan Co., Seoul, Korea) and the remaining six persons consumed $240 \mathrm{~mL}$ of blueberry juice (total polyphenols $300 \mathrm{mg}$ and proanthocyanidin $76 \mathrm{mg} / \mathrm{d}$ ) for 2 weeks. Contents of blueberry juice were measured by Korea Health Supplement Institute. Due to discontinuity, two subjects of the vitamin $C$ group were excluded (Fig. 1).

The first voids of urine before breakfast were collected at 0,4 , 7, and 14 days from the first intake. The urine samples were stored at $-20^{\circ} \mathrm{C}$ until analysis. Blood samples $(10 \mathrm{~mL})$ were collected by venipuncture into the evacuated tubes containing sodium heparin as an anticoagulant (BD Vacutainer, Franklin Lakes, NJ, USA) before and after the trial. Questionnaire data, regarding physical status, lifestyle, dietary patterns, and contents during the intervention period, were obtained from the study

A

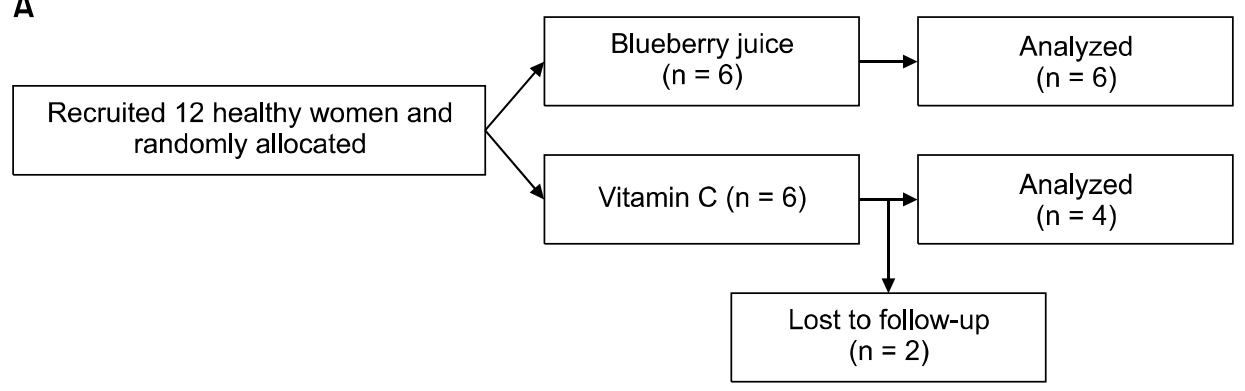

B

\begin{tabular}{c|cccc|}
\multicolumn{1}{c}{} & Day 0 & 4 & 7 & 14 \\
\cline { 2 - 5 } Urine & $\sqrt{ }$ & $\sqrt{ }$ & $\sqrt{ }$ & $\sqrt{ }$ \\
Blood & $\sqrt{ }$ & & & $\sqrt{ }$ \\
\cline { 2 - 4 } & & & &
\end{tabular}

Blueberry juice or vitamin C
Figure 1. The study schedule. (A) The allocation of subjects. (B) The collection spots of biospecimens $(\sqrt{ })$. 
participants. Quality-controlled fresh juice of blueberry (120 $\mathrm{mL} /$ bottle) was provided daily by Daesang Well Life Co. (Seoul, Korea) and consumed within 24 hours. This study was approved by Institutional Review Board of Sookmyung Women's University (SMWU-1310-BR-009-002).

\section{Analysis of 8-hydroxydeoxyguanosin}

The urinary 8-OHdG levels were determined based on the automated two-step separation method. ${ }^{11}$ Briefly, each defrosted urine sample was centrifuged at 13,000 rpm for 5 minutes and 50 $\mu \mathrm{L}$ urine supernatant was mixed with the same volume of a dilution solution containing the ribonucleoside marker 8-hydroxyguanosine. A $20 \mu \mathrm{L}$ aliquot of the diluted urine sample was injected to an automated high-performance liquid chromatography (HPLC) system. The anion exchange guard column (MCI GEL CA08F, $1.5 \times 150$ mm; Mitsubishi Chemical, Tokyo, Japan) was used in HPLC-1. The chromatograms were recorded by a Gilson UV detector (UV/VIS-151, $235 \mathrm{~nm}$ ). The 8-OHdG fraction was collected, automatically injected into a reverse-phase column in HPLC-2 (Capcell Pak C18, $4.6 \times 250$ mm; GL Sciences Inc., Tokyo, Japan), and detected by an EC detector (Coulochem II; ESA, Chelmsford, MA, USA). 8-OHdG levels were normalized for urinary creatinine levels. Urinary creatinine was simultaneously analyzed with UV detector at $225 \mathrm{~nm}$ to adjust individual urine density.

\section{Analyses of malondialdehyde}

MDA was determined by the level of adducts with 2-thiobarbituric acid (CAS number: 504-17-6) as our previously described method with the same HPLC-system. ${ }^{12,13}$

4. Quantification of DNA methylation at DNA methyltransferase 1, NAD $(\mathrm{P}) \mathrm{H}$ quinone oxidoreductase 1 , and methylenetetrahydrofolate reductase genes

Genomic DNA was isolated from the peripheral blood with the QIAamp DNA Blood Mini kit (QIAGEN, Valencia, CA, USA), following the manufacturer's instructions. The purity and concentration of the isolated genomic DNA were measured using the NanoDrop ND-1000 spectrophotometer (NanoDrop Technologies, Wilmington, DE, USA). Genomic DNA samples were digested with the EpiTect Methyl DNA Restriction Kit (QIAGEN). Real-time PCR was performed with $12.5 \mu \mathrm{L}$ of $\mathrm{RT}^{2}$ SYBR Green ROX qPCR Mastermix (QIAGEN), $5 \mu \mathrm{L}$ of the digested DNA (Mo, $\mathrm{Ms}, \mathrm{Md}$, or Msd), $1.0 \mu \mathrm{L}$ of primers (final concentration, $25 \mathrm{pmol}$ ) and $6.5 \mu \mathrm{L}$ of RNase-/DNase-free water on the ABI7500 (Applied Biosystems, Foster City, CA, USA). The primers for DNMT1,
NQO1, and MTHFR were purchased from QIAGEN: EPHS107019-1A (CpG Island\# 107019), MePH28268-1A (CpG Island\# 105427), and EPHS115460-1A (CpG Island\# 115460), respectively. The $\mathrm{PCR}$ reactions were carried out using the following conditions: 1 cycle at $95^{\circ} \mathrm{C}$ for 10 minutes, 3 cycles at $99^{\circ} \mathrm{C}$ for 30 seconds and $72^{\circ} \mathrm{C}$ for 1 minute, 40 cycles at $97^{\circ} \mathrm{C}$ for 15 seconds and $72^{\circ} \mathrm{C}$ for 1 minute. A dissociation stage was added between $60^{\circ} \mathrm{C}$ and $95^{\circ} \mathrm{C}$. Percentages of methylated DNA were calculated by the QIAGEN-provided software.

\section{Analysis of global DNA methylation}

We analyzed the global DNA methylation levels in each genomic DNA sample with the MethylFlash Methylated DNA Quantification kit (EpigenTek, Brooklyn, NY, USA), based on the ELISA method, following the manufacturer's protocol. Briefly, $100 \mathrm{ng}$ of each genomic DNA was bound to strip wells. The 5-methylcytosine (5-mC) of the DNA samples was reacted with the specific antibody. The absorbance of the antigen-antibody-complex was analyzed at $450 \mathrm{~nm}$ using a EL $\times 800$ Microplate spectrophotometer (Bio-Tek, Winooski, VT, USA). The amount of 5-mC (\%) was quantified from a standard curve $(0,0.5,1.0,2.0,5.0$, and $10.0 \mathrm{ng})$.

\section{Analysis of the methylenetetrahydrofolate reductase genetic polymorphism}

Genetic polymorphism of the MTHFR (rs1801133, C677T in exon 4) was studied by PCR-RFLP analysis. ${ }^{14}$ Briefly, genomic DNA was amplified by PCR in the GeneAmp PCR system 2700 (Applied Biosystems). The PCR mixture $(20 \mu \mathrm{L})$ was prepared to contain 20 ng of DNA template, $10 \mathrm{pmol}$ of each primer, and RNase-/DNase-free water in the AccuPower PCR PreMix (Bioneer Co., Daejeon, Korea). After confirming the PCR products by electrophoresis, $10 \mu \mathrm{L}$ of the DNA products was digested with 10 units of Hinf1 (Takara Biomedicals, Shiga, Japan) at $37^{\circ} \mathrm{C}$ overnight. The Hinf1-treated PCR fragments were analyzed by electrophoresis with 4\% Metaphor agarose gel.

\section{Statistical analysis}

Shapiro-Wilk test was used to test the distributional normality in levels of urinary 8-OHdG and MDA and \% of global/specific DNA methylation. T-test or Pearson's test were used to study the differences in characteristics between the blueberry juice and the vitamin $\mathrm{C}$ groups before the intervention.

Pearson product-moment correlation ( $\mathrm{r}$ ) was used to analyze the correlation between levels of urinary 8-OHdG and MDA, and to find effects of the treatment on the above two oxidative 
biomarkers during the trial. Kinetic changes in oxidative stress biomarkers 8-OHdG and MDA were compared between the

Table 1. Characteristics of food intake in study subjects

\begin{tabular}{|c|c|c|c|}
\hline Food intake & $\begin{array}{l}\text { Blueberry } \\
\text { group (n) }\end{array}$ & $\begin{array}{l}\text { Vitamin C } \\
\text { group }(\mathrm{n})\end{array}$ & $P$-value \\
\hline Dietary balance & & & 0.75 \\
\hline Preference for meat & 1 & 1 & \\
\hline Balanced diet & 5 & 3 & \\
\hline Preference for vegetables & 0 & 0 & \\
\hline Yogurt & & & 0.43 \\
\hline$<$ Once/mo & 0 & 0 & \\
\hline 1-2 times/mo & 2 & 1 & \\
\hline $1-2$ times/wk & 4 & 2 & \\
\hline Everyday & 0 & 1 & \\
\hline Kimchi $^{\mathrm{a}}$ & & & 0.52 \\
\hline$<$ Once/wk & 0 & 0 & \\
\hline 1-2 times/wk & 1 & 2 & \\
\hline 3-4 times/wk & 3 & 1 & \\
\hline Everyday & 2 & 1 & \\
\hline Alcohol & & & 0.33 \\
\hline$<$ Once/mo & 2 & 1 & \\
\hline $1-2$ times $/ \mathrm{mo}$ & 2 & 3 & \\
\hline $1-2$ times/wk & 2 & 0 & \\
\hline$\geq 3-4$ times/wk & 0 & 0 & \\
\hline Coffee & & & 0.41 \\
\hline$<$ Once/wk & 1 & 1 & \\
\hline 1-2 times/wk & 1 & 2 & \\
\hline Everyday & 4 & 1 & \\
\hline Instant noodles (ramyeon) & & & 0.33 \\
\hline$<$ Once/mo & 1 & 2 & \\
\hline $1-2$ times $/ \mathrm{mo}$ & 3 & 2 & \\
\hline $1-2$ times/wk & 2 & 0 & \\
\hline$\geq 3-4$ times/wk & 0 & 0 & \\
\hline
\end{tabular}

${ }^{\mathrm{a}} \mathrm{A}$ fermented side dish made of vegetables with a variety of seasonings.

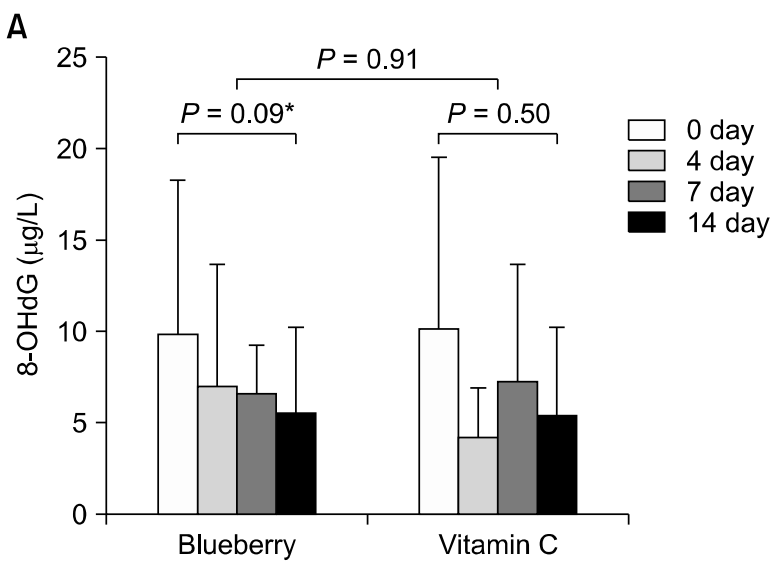

blueberry juice and the vitamin $C$ groups by repeated ANOVA (MANOVA) during the trial (2 weeks).

Wilcoxon rank- sum test was used to analyze the significances of $\Delta$ of 8-OHdG or $\Delta$ of MDA levels, i.e., [8-OHdG]before $[8-\mathrm{OHdG}]_{\text {after }}$ or $[\mathrm{MDA}]_{\text {before }}-[\mathrm{MDA}]_{\text {after }}$, respectively, in each (blueberry or vitamin C) group, the significances of $\Delta \Delta$ of 8-OHdG or MDA levels, i.e., $\Delta$ of $[8-\mathrm{OHdG}]_{\mathrm{vtc}}-\Delta$ of $[8-\mathrm{OHdG}]_{b l u e b e r y}$ or $\triangle$ of $[\mathrm{MDA}]_{\mathrm{vtC}}-\triangle$ of $[\mathrm{MDA}]_{\text {bluebery }}$, respectively and the significances of $\triangle$ or $\triangle \triangle$ of MTHFR hypermethylation by 'vitamin $C$ or blueberry consumption' due to the susceptibility to MDA.

Regression analyses were used to study the correlation between consumption periods of blueberry or vitamin $\mathrm{C}$ and levels of urinary 8-OHdG, MDA, or DNA methylation. Statistical significance was considered at $P<0.05$. The JMP ver. 4 (SAS Institute, Cary, NC, USA) was used for all statistical analyses.

\section{RESULTS}

\section{Characteristics of the subjects}

All subjects were healthy non-smoking young women. Body mass index of the subjects $\left(20.0 \pm 2.0 \mathrm{~kg} / \mathrm{m}^{2}\right)$ was within the normal range for young Korean women. There were no statistically significant differences in the characteristics, particularly food intake, of subjects between the blueberry and the vitamin C groups before the intervention (Table 1). Thus, the appropriateness of our randomization for the intervention was confirmed.

\section{No differences in anti-oxidative effects between blueberry and vitamin $C$ consumption}

The ranges of urinary 8-OHdG and MDA were 0.64 to 25.18

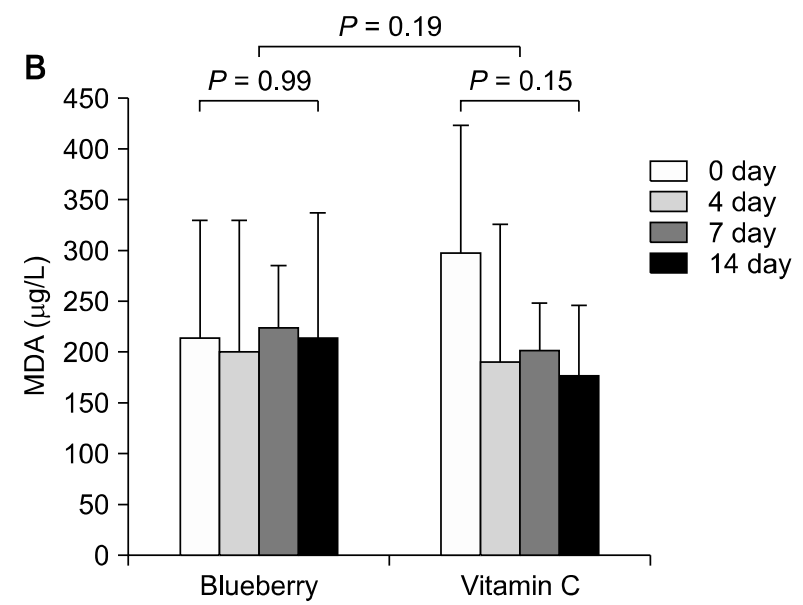

Figure 2. Comparison of oxidative biomarkers between before (day 0 ) and after (day 14) the intervention. (A) 8-hydroxydeoxyguanosin (8-OHdG); (B) malondialdehyde (MDA). ${ }^{*} 0.05<P<0.1$ (Wilcoxon rank-sum test). 
$\mathrm{ng} / \mathrm{mL}$ and 8.17 to $493.82 \mathrm{ng} / \mathrm{L}$, respectively. Urinary 8-OHdG did not follow the normal distribution; however, urinary MDA followed ( $P<0.05$ and $P=0.05$ by Shapiro-Wilk test). There was a positive association between the two biomarkers $(\mathrm{r}=0.67, P<$ 0.01 by regression analysis). The average levels of urinary 8-OHdG and MDA were reduced during the intervention, however, their reductions were not significant ( $P=0.17$ and 0.37 , respectively). In addition, there were no significant differences in the two biomarkers due to the treatments ( $P=0.78$ for urinary 8-OHdG; $P=0.49$ for urinary MDA by MANOVA). Blueberry seems to have similar antioxidative effects to vitamin $\mathrm{C}$.

In detail, borderline-significant reduction of urinary 8-OHdG levels was observed on the 14 day of blueberry consumption $(P=$ 0.09 ; Fig. $2 \mathrm{~A})$, but not vitamin $C$ consumption $(P=0.50)$. However, no significant differences were found in blueberry-reduced urinary 8-OHdG levels by relative comparison with vitamin $C$ ( $P=0.91$; Fig. 2A).

In a case of urinary MDA, no significant changes were found by the blueberry juice or vitamin $\mathrm{C}$ consumption (Fig. 2B). In detail, there was somewhat decrease of urinary MDA levels in the vitamin $C$ group, not in the blueberry group $(P=0.15$ for the vitamin C vs. $P=0.99$ for the blueberry). However, there was no significant difference in the relative changes of urinary MDA levels between the two groups on the 14 day ( $P=0.19$; Fig. 2B). Therefore, antioxidative effects of blueberry and vitamin $C$ were confirmed to be similar, as they were measured with the two different oxidative stress biomarkers.

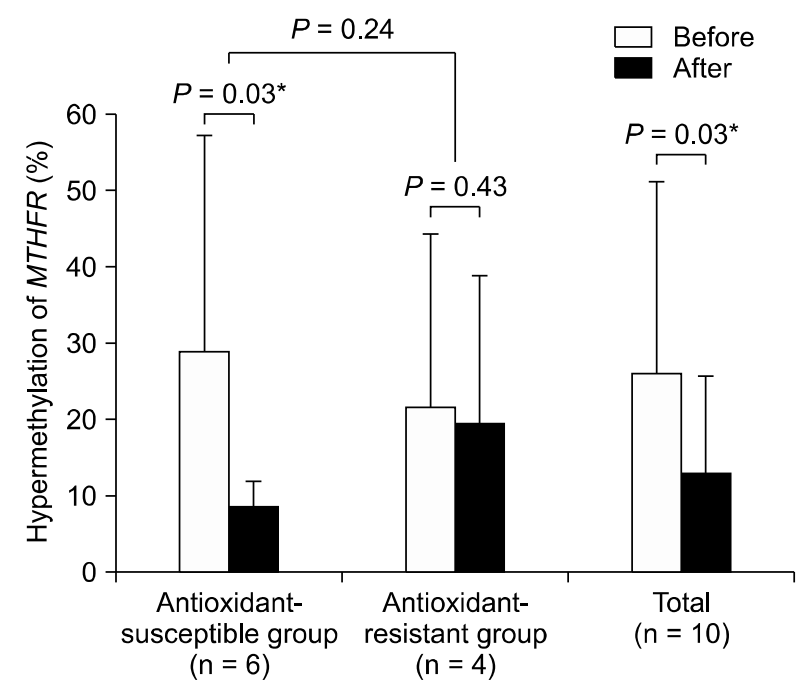

Figure 3. Reduced methylenetetrahydrofolate reductase (MTHFR) hypermethylation by vitamin $C$ or blueberry: Total or the antioxidant-susceptible subjects showed significant decrease of the MTHFR hypermethylation $(* P<0.05)$.

\section{Effects of blueberry juice consumption on specific and global DNA methylation}

Individual variations in susceptibility to vitamin $\mathrm{C}$ or blueberry were further studied. We found that most subjects showed the decreased urinary 8-OHdG after the trial. However, urinary MDA levels were decreased in $60 \%(n=6)$ of all subjects after the trial. Therefore, the six subjects were defined as the antioxidant susceptible subjects and the others as the antioxidant resistant subjects. Interestingly, hypermethylation (\%) of the MTHFR was significantly decreased by the blueberry or vitamin $C$ consumption $(P=0.03$; Fig. 3$)$ in the antioxidant-susceptible subgroup ( $\mathrm{n}=6$ including 3 blueberry consumers and 3 vitamin $C$ consumers). In all subjects ( $n=10)$, the MTHFR methylation was decreased by blueberry juice or vitamin $C$ intake ( $P=0.03$ by Wilcoxon-test; Fig. 3).

When separated the vitamin $C$ and the blueberry group, the blueberry group had high levels of MTHFR hypermethylation before the trial and showed big reduction of the MTHFR hypermethylation (Fig. 4).

Epigenetic changes in global or other specific genes were also studied. As results, no significant effects of blueberry juice or vitamin $\mathrm{C}$ consumption were found on the promoter methylation of $D N M T 1$ or $N Q O 1$ ( $P=0.62$ or $P=0.56$, respectively) or global methylation $(P=0.47)$.

\section{Interaction between epigenetic and anti-oxidative effects of blueberry}

Among specific genes, we found that the changes of urinary 8-OHdG were positively correlated with those of DNA methylation at the MTHFR and the DNMT1 $(P<0.05)$ rather than other

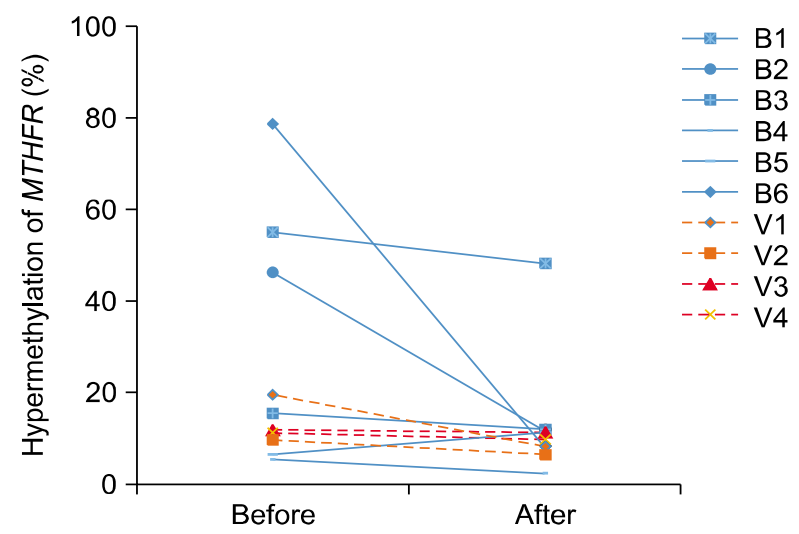

Figure 4. Repressed DNA hypermethylation at the promoter region of the methylenetetrahydrofolate reductase (MTHFR) by the intervention. B1-6, blueberry consumers; V1-4, vitamin C consumers. 
genes (Fig. 5A). However, the changes of urinary MDA were not significantly associated with those of any epigenetic marker (Fig. $5 \mathrm{~B})$. Among the genes, there was a positive association between the changes of DNA methylation at the MTHFRand the NQOI ( $P$ $<0.01$ ). Therefore, epigenetic modulation of blueberry juice or vitamin $\mathrm{C}$ may be related to interaction of the above two genes.

\section{Effects of the methylenetetrahydrofolate reductase polymorphism on antioxidative and epigenetic capacity of blueberry or vitamin C}

By genotyping of the MTHFR polymorphism (C677T), five subjects were diagnosed to be the CC, four to be the CT, and one to be the TT genotype. The frequency of the mutant (T) allele was 0.3. No significant effects of the MTHFR polymorphisms were found on urinary levels of 8-OHdG or MDA, or methylation levels of the MTHFR or DNMT1 $(P>0.05)$.

\section{DISCUSSION}

Chemopreventive effects of blueberry, particularly its antioxidative function, are obscure in human. ${ }^{15}$ It may be related to differences in measuring biomarkers for ROS, amounts of blueberry intake, or characteristics of the subjects. Therefore, we simultaneously analyzed two oxidative biomarkers, 8-OHdG and MDA, to evaluate the antioxidative effects of blueberry. The urinary 8-OHdG levels were marginally decreased by blueberry juice consumption, whereas the urinary MDA level was not (Fig. 2). Others reported that serum oxygen radical absorbance capacity (ORAC), rather than serum lipoprotein oxidation, was significantly higher in the blueberry group than in the control group. ${ }^{15}$ Therefore, blueberry may show antioxidative effects on global oxidative stress biomarkers, e.g., 8-OHdG or ORAC, rather than lipid peroxidation biomarkers, e.g., MDA.

Other study showed that the lipid peroxidation levels, i.e., MDA levels, were significantly decreased by vitamin $\mathrm{C}$ consumption. ${ }^{16}$ However, some studies showed vitamin $\mathrm{C}$ consumption had no significant effect on oxidative DNA damage as demonstrated by urinary $8-0 H d G$ levels. ${ }^{17}$ In the present study, the antioixdative effects of vitamin $\mathrm{C}$ were somewhat shown in urinary MDA rather than 8-OHdG (Fig. 3). Therefore, our results suggest that blueberry and vitamin $\mathrm{C}$ may have different mechanisms for the antioxidative effects or different sensitivities to different oxidative stress-related biomarkers.

The amount of blueberry or food intake may affect its antioxidative effects in human. Blacker et al. ${ }^{15}$ showed that ORAC was increased with small dose of blueberry $(75 \mathrm{~g})$ at 1 and 2 hours after the blueberry consumption with a high-carbohydrate and low-fat breakfast and suggested that a smaller dose of blueberry could provide increase in serum antioxidant capacity. However, Prior et al. ${ }^{18}$ reported a significant increase in serum antioxidant capacity at 1 and 2 hours post-consumption of high dose (189 g), not low dose $(94.5 \mathrm{~g})$, of frozen blueberry without any additional feeding. In the present study, we used the volume commonly consumed ( $240 \mathrm{~mL}$ of blueberry juice $\fallingdotseq 70 \mathrm{~g}$ of blueberry) and recorded daily food intake (items and amounts). Most of the present subjects consumed balanced diet of carbohydrate, protein, and fat during the trial. Therefore, our results represented relatively small volume of blueberry with well
A

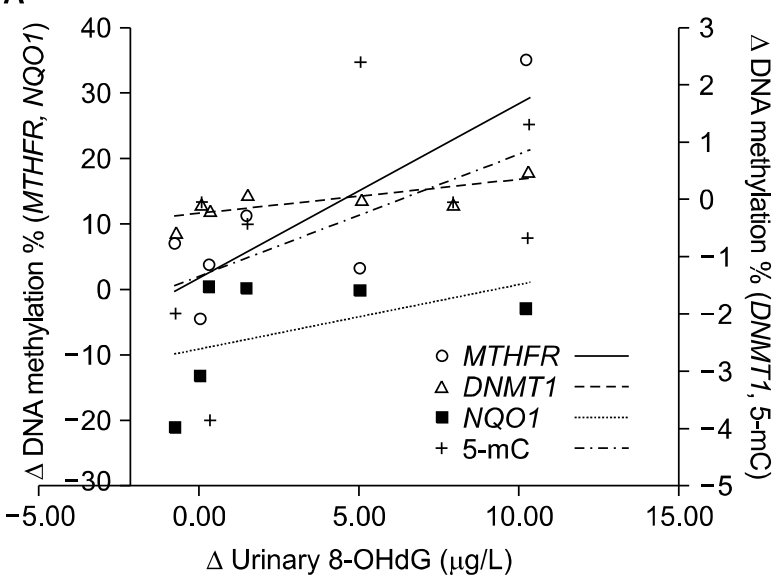

B

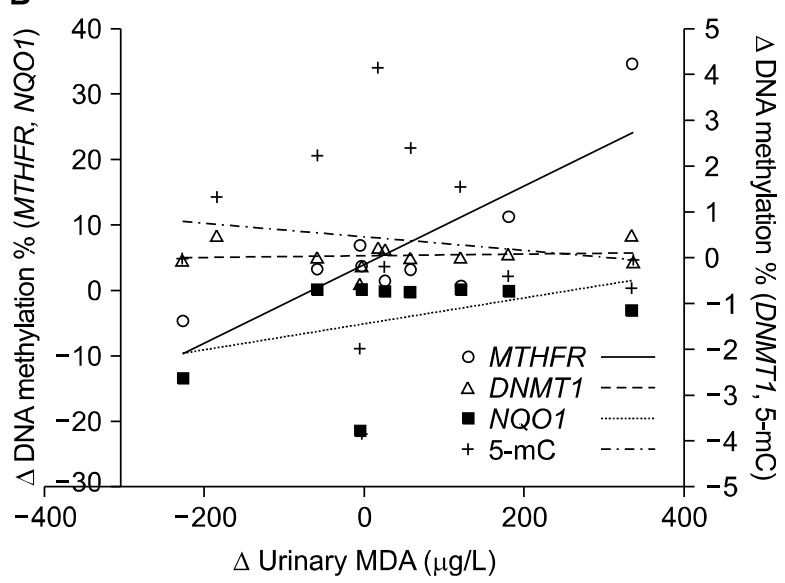

Figure 5. Relationship between epigenetic and antioxidative changes by the consumption of blueberry juice or vitamin C. (A) $\triangle$ urinary 8-OHdG $=[\text { urinary 8-OHdG }]_{\text {before }}-[\text { urinary 8-OHdG }]_{\text {after, }} \Delta$ DNA methylation $=[\text { DNA methylation }]_{\text {before }}-[\text { DNA methylation }]_{\text {after. }}$ (B) $\Delta$ urinary $\mathrm{MDA}=[\text { urinary MDA }]_{\text {before }}-$ [urinary $\left.8 \mathrm{MDA}\right]_{\text {after. }}$ MTHFR, methylenetetrahydrofolate reductase; NQO1, NAD(P)H quinone oxidoreductase 1; DNMT1, DNA methyltransferase 1; 5-mC, 5-methylcytosine; 8-OHdG, hydroxydeoxyguanosine; MDA, malondialdehyde. 
balanced diet, compared to the previous blueberry studies. ${ }^{15,18}$

Lastly, characteristics of the subjects can be considered. In the present study, we recruited healthy, nonsmoking, and young women. It is a severe condition to evaluate antioxidative effects of blueberry, compared to that of sick or elder people. ${ }^{19}$ Practically, urinary 8-OHdG or MDA levels in the present subjects are relatively low, compared to others, such as smokers and unhealthy individuals. ${ }^{20}$ Even vitamin $\mathrm{C}$, a positive control for antioxidative agents, did not show significant antioxidative effects in the present study (Fig. 2). That is, it is hard to find significant effects of antioxidative agents among normal subjects.

The MTHFR is involved in the metabolism of folate, a well-known epigenetic modifier, and affects DNA synthesis and conversion of homocysteine to methionine. ${ }^{1}$ DNA methylation at the promoter sequence of the MTHFR is a candidate for down-regulation of the MTHFR expression. ${ }^{21}$ In addition, aberrant hypermethylation of the MTHFR was reported in cancers of the upper aerodigestive tract. ${ }^{22}$ The reduced expression of the MTHFR increases the risk of cancer, e.g., oesophageal squamous cell carcinoma. ${ }^{23}$ Recently, re-expression of abnormally silenced suppressor genes is being investigated for potential clinical applications. ${ }^{24}$ Likewise, most subjects in the present study showed decreased hypermethylation (\%) of the MTHFR after the blueberry juice or vitamin $C$ treatment (Fig. 3 and 4). Therefore, the present study suggests that potential anticancer mechanism of blueberry or vitamin $C$ is associated with inhibition of promoter methylation at the MTHFR.

Association between oxidative stress and epigenetic alterations has been extensively studied to understand the pathogenesis of oxidative stress-related diseases. ${ }^{3.25}$ For example, the generation of ROS and 8-OHdG could play a critical role in the abnormal methylation of tumor suppressor genes in the liver of chronic hepatitis C. Nishida et al. ${ }^{3}$ demonstrated that the 8-OHdG levels were associated with increased numbers of methylated tumor suppressors, and that tumor suppressor genes carrying higher levels of 8-OHdG facilitated the modification of the active chromatin to a repressive form after stimulation by ROS. In addition, the methylation of a tumor suppressor gene, e.g., runt-related transcription factor 3 , was significantly increased in bladder cancer cells exposed to $\mathrm{H}_{2} \mathrm{O}_{2}$. However, it was markedly decreased in cells pretreated with an antioxidant, tocopheryl acetate. ${ }^{25}$ The present study showed that the changes of urinary 8-OHdG were positively associated with those of DNA methylation at the MTHFR and the DNMT1 by 'vitamin C- or blueberry consumption' (Fig. 5A). Therefore, it suggests that antioxidants may exert their biological effect through modula- tion of DNA methylation.

Additionally, we found a positive association between the changes of DNA methylation at the MTHFR and the NQO1 in the present study. Disruption of the $N Q O 1$ has been shown to alter intracellular redox status and result in excessive ROS production leading to toxicity, mutations, and ultimately development of cancer in various human tissues. For example, hepatitis B virus-induced epigenetic silencing of the $\mathrm{NQO1}$ increased mitochondrial injury and cellular susceptibility to oxidative stress in hepatoma cells. ${ }^{26}$ In addition, alpha-lipoic acid was reported to protect from carcinogenic chemical-induced lipid peroxidation and its protective effects were mediated by the induction of $N Q O^{27}$ Moreover, inactivation of the MTHFR induced hyperhomocysteinemia and oxidative stress. ${ }^{28}$ Therefore, these findings support that blueberry- or vitamin C-induced antioxidative actions may occur via epigenetic modulation on the MTHFR and NQO1. In conclusion, blueberry juice has similar anti-oxidative or anti-premutagenic activity to vitamin $\mathrm{C}$ and the potential of methylation inhibitors for the MTHFR and the $D N M T 1$ in human.

\section{ACKNOWLEDGMENTS}

This study was supported by the 'Health Fellowship Foundation' -grant and the National Research Foundation of Korea (NRF) funded by the Korea government (MISP) (No. 2011-0030074). We deeply appreciate Dr. Sukkil Rose Koh for her comments and revise of English.

\section{CONFLICTS OF INTEREST}

No potential conflicts of interest were disclosed.

\section{REFERENCES}

1. Kim M, Bae M, Na H, Yang M. Environmental toxicants: induced epigenetic alterations and their reversers. J Environ Sci Health $\mathrm{C}$ Environ Carcinog Ecotoxicol Rev 2012;30:323-67.

2. Haggarty P. Epigenetic consequences of a changing human diet. Proc Nutr Soc 2013;72:363-71.

3. Nishida N, Arizumi T, Takita M, Kitai S, Yada N, Hagiwara S, et al. Reactive oxygen species induce epigenetic instability through the formation of 8-hydroxydeoxyguanosine in human hepatocarcinogenesis. Dig Dis 2013;31:459-66.

4. Bernal AJ, Dolinoy DC, Huang D, Skaar DA, Weinhouse C, Jirtle RL. Adaptive radiation-induced epigenetic alterations mitigated by antioxidants. FASEB J 2013;27:665-71.

5. Yi B, Kasai H, Lee HS, Kang Y, Park JY, Yang M. Inhibition by wheat sprout (Triticum aestivum) juice of bisphenol A-induced 
oxidative stress in young women. Mutat Res 2011;724:64-8.

6. Speciale A, Cimino F, Saija A, Canali R, Virgili F. Bioavailability and molecular activities of anthocyanins as modulators of endothelial function. Genes Nutr 2014:9:404.

7. Suh N, Paul S, Hao X, Simi B, Xiao H, Rimando AM, et al. Pterostilbene, an active constituent of blueberries, suppresses aberrant crypt foci formation in the azoxymethane-induced colon carcinogenesis model in rats. Clin Cancer Res 2007;13:350-5.

8. Moriwaki T, Kato S, Kato Y, Hosoki A, Zhang-Akiyama QM. Extension of lifespan and protection against oxidative stress by an antioxidant herb mixture complex (KPG-7) in Caenorhabditis elegans. J Clin Biochem Nutr 2013;53:81-8.

9. Baba AB, Kowshik J, Krishnaraj J, Sophia J, Dixit M, Nagini S. Blueberry inhibits invasion and angiogenesis in 7,12-dimethylbenz[a]anthracene (DMBA)-induced oral squamous cell carcinogenesis in hamsters via suppression of TGF- $\beta$ and NF-KB signaling pathways. J Nutr Biochem 2016;35:37-47.

10. Qi C, Li S, Jia Y, Wang L. Blueberry anthocyanins induce G2/M cell cycle arrest and apoptosis of oral cancer $\mathrm{KB}$ cells through down-regulation methylation of p53. Yi Chuan 2014;36:566-73.

11. Kasai H. A new automated method to analyze urinary 8-hydroxydeoxyguanosine by a high-performance liquid chromatography-electrochemical detector system. J Radiat Res 2003:44:185-9.

12. Kho MJ, Bae MJ, Yang M. Chemopreventive effects of Korean red ginseng on urinary bisphenol $\mathrm{A}$ and malondialdehyde. Cancer Prev Res 2010;15:231-5.

13. Yang M, Lee HS, Hwang MW, Jin M. Effects of Korean red ginseng (Panax Ginseng Meyer) on bisphenol A exposure and gynecologic complaints: single blind, randomized clinical trial of efficacy and safety. BMC Complement Altern Med 2014;14:265.

14. Frosst P, Blom HJ, Milos R, Goyette P, Sheppard CA, Matthews RG, et al. A candidate genetic risk factor for vascular disease: a common mutation in methylenetetrahydrofolate reductase. Nat Genet 1995;10:111-3.

15. Blacker BC, Snyder SM, Eggett DL, Parker TL. Consumption of blueberries with a high-carbohydrate, low-fat breakfast decreases postprandial serum markers of oxidation. Br J Nutr 2013;109: 1670-7.

16. Candan F, Gültekin F, Candan F. Effect of vitamin C and zinc on osmotic fragility and lipid peroxidation in zinc-deficient haemodialysis patients. Cell Biochem Funct 2002;20:95-8.

17. Huang HY, Helzlsouer KJ, Appel LJ. The effects of vitamin $C$ and vitamin $\mathrm{E}$ on oxidative DNA damage: results from a randomized controlled trial. Cancer Epidemiol Biomarkers Prev 2000;9: 647-52.

18. Prior RL, Cao G, Prior RL, Cao G. Analysis of botanicals and dietary supplements for antioxidant capacity: a review. J AOAC Int 2000;83:950-6.

19. Johnson SA, Feresin RG, Navaei N, Figueroa A, Elam ML, Akhavan NS, et al. Effects of daily blueberry consumption on circulating biomarkers of oxidative stress, inflammation, and antioxidant defense in postmenopausal women with pre-and stage 1-hypertension: a randomized controlled trial. Food Funct 2017;8:372-80.

20. Agarwal R. Smoking, oxidative stress and inflammation: impact on resting energy expenditure in diabetic nephropathy. BMC Nephrol 2005;6:13.

21. Khazamipour N, Noruzinia M, Fatehmanesh P, Keyhanee M, Pujol P. MTHFR promoter hypermethylation in testicular biopsies of patients with non-obstructive azoospermia: the role of epigenetics in male infertility. Hum Reprod 2009:24:2361-4.

22. Mani S, Szymańska K, Cuenin C, Zaridze D, Balassiano K, Lima $\mathrm{SC}$, et al. DNA methylation changes associated with risk factors in tumors of the upper aerodigestive tract. Epigenetics 2012;7: 270-7.

23. Liu R, Yin L, Pu Y. Association between gene expression of metabolizing enzymes and esophageal squamous cell carcinomas in China. Genet Test Mol Biomarkers 2012;16:1211-7.

24. Ushijima M, Ogata Y, Tsuda H, Akagi Y, Matono K, Shirouzu K. Demethylation effect of the antineoplaston AS2-1 on genes in colon cancer cells. Oncol Rep 2014:31:19-26.

25. Wongpaiboonwattana W, Tosukhowong P, Dissayabutra T, Mutirangura A, Boonla C. Oxidative stress induces hypomethylation of LINE-1 and hypermethylation of the RUNX3 promoter in a bladder cancer cell line. Asian Pac J Cancer Prev 2013;14:3773-8.

26. Wu YL, Wang D, Peng XE, Chen YL, Zheng DL, Chen WN, et al. Epigenetic silencing of NAD P)H:quinone oxidoreductase 1 by hepatitis $B$ virus $X$ protein increases mitochondrial injury and cellular susceptibility to oxidative stress in hepatoma cells. Free Radic Biol Med 2013;65:632-44.

27. Podder B, Kim YS, Song HY. Cytoprotective effect of bioactive sea buckthorn extract on paraquat-exposed A549 cells via induction of Nrf2 and its downstream genes. Mol Med Rep 2013;8:1852-60.

28. Lemarié CA, Shbat L, Marchesi C, Angulo OJ, Deschênes ME, Blostein MD, et al. Mthfr deficiency induces endothelial progenitor cell senescence via uncoupling of eNOS and downregulation of SIRT1. Am J Physiol Heart Circ Physiol 2011;300:H745-53. 